\title{
Prescribed conditions at infinity for fractional parabolic and elliptic equations with unbounded coefficients
}

\author{
Fabio Punzo* and Enrico Valdinoci ${ }^{\dagger}$
}

\begin{abstract}
We investigate existence and uniqueness of solutions to a class of fractional parabolic equations satisfying prescribed pointwise conditions at infinity (in space), which can be timedependent. Moreover, we study the asymptotic behaviour of such solutions. We also consider solutions of elliptic equations satisfying appropriate conditions at infinity.
\end{abstract}

\section{Introduction}

We are concerned with existence and uniqueness of solutions to the following linear nonlocal parabolic Cauchy problem:

$$
\begin{cases}\partial_{t} u=-a(-\Delta)^{s} u+c u+f & \text { in } \mathbb{R}^{N} \times(0, T]=: S_{T} \\ u=u_{0} & \text { in } \mathbb{R}^{N} \times\{0\},\end{cases}
$$

where the coefficient $a$ is a positive function only depending on the space variable $x$, which becomes unbounded as $|x| \rightarrow \infty ;(-\Delta)^{s}$ denotes the fractional Laplace operator of order $s \in$ $(0,1), N>2 s$, while $c, f, u_{0} \in L^{\infty}\left(\mathbb{R}^{N}\right)$. Moreover, we investigate existence and uniqueness of solutions to the linear nonlocal elliptic equation

$$
a(-\Delta)^{s} u-c u=f \quad \text { in } \mathbb{R}^{N}
$$

in this case we also suppose that $c \leq 0$.

(a) Parabolic problems. The well-posedness of problem (1.1) has been largely studied in the literature in the local case $s=1$ (see, e.g., [2], [8, [12, [15, [16, [17, 18, [22, 28]) . As a matter of fact, if $N=1,2$ and $s=1$, then there exists a unique bounded solution of problem (1.1). If $N \geq 3$, a special role is played by the behaviour at infinity of the coefficient a. In particular, if

$$
a(x) \leq C\left(1+|x|^{2}\right)^{\frac{\alpha}{2}} \quad \text { for all } x \in \mathbb{R}^{N} \text {, for some } C>0, \alpha \leq 2,
$$

\footnotetext{
*Dipartimento di Matematica "Federigo Enriques", Università degli Studi di Milano, via Cesare Saldini 50, 20133 Milano, Italy (fabio.punzo@unimi.it).

${ }^{\dagger}$ Weierstraß Institut für Angewandte Analysis und Stochastik, Mohrenstraße 39, 10117 Berlin, Germany, and Dipartimento di Matematica "Federigo Enriques", Università degli Studi di Milano, Via Cesare Saldini 50, 20133 Milano, Italy, and Istituto di Matematica Applicata e Tecnologie Informatiche "Enrico Magenes", Consiglio Nazionale delle Ricerche, Via Ferrata 1, 27100 Pavia, Italy (enrico@math.utexas.edu). Supported by the ERC grant $\epsilon$ (Elliptic Pde's and Symmetry of Interfaces and Layers for Odd Nonlinearities). Both authors are supported by PRIN grant 201274FYK7 (Critical Point Theory and Perturbative Methods for Nonlinear Differential Equations).
} 
then problem (1.11) admits only one bounded solution (see [2], [15]). Instead, if

$$
a(x) \geq C\left(1+|x|^{2}\right)^{\frac{\alpha}{2}} \text { for all } x \in \mathbb{R}^{N} \text {, for some } C>0, \alpha>2,
$$

then problem (1.1) admits infinitely many bounded solutions. More precisely, for any given $g \in C([0, T])$, if

$$
\lim _{|x| \rightarrow \infty} u_{0}(x)=g(0)
$$

then there exists a unique bounded solution of problem (1.1) such that

$$
\lim _{|x| \rightarrow \infty} u(x, t)=g(t) \quad \text { uniformly with respect to } t \in[0, T]
$$

(see [12, 18]). Observe that condition (1.4) can be regarded as a Dirichlet condition at infinity, which is time-dependent.

More recently, existence and uniqueness results for nonlocal Cauchy parabolic problems have been established. In this respect, in [1, [19], 20] a quite general class of integrodifferential equations have been treated; it also includes problem (1.1) if there exist two constants $C_{1}>0, C_{2}>0$ such that

$$
C_{1} \leq a(x) \leq C_{2} \text { for all } x \in \mathbb{R}^{N} .
$$

Furthermore, the well-posedness for the Cauchy problem associated to the fractional porous medium equation with a variable density $a=a(x)$ has been studied in [13, 14, 23], allowing that $a(x) \rightarrow \infty$ or $a(x) \rightarrow 0$ as $|x| \rightarrow \infty$. Moreover, in 25] the uniqueness of solutions of problem (1.1) with $c \equiv 0$ in suitable weighted Lebesgue spaces is stated. To be more specific, let

$$
\psi(x):=\left(1+|x|^{2}\right)^{-\frac{\beta}{2}} \quad\left(x \in \mathbb{R}^{N}\right),
$$

$\beta$ being a positive parameter. Suppose that, for some $C>0$ and $\alpha \in \mathbb{R}$,

$$
a(x) \leq C\left(1+|x|^{2}\right)^{\frac{\alpha}{2}} \quad(x \in \mathbb{R}) .
$$

Let $p \geq 1$, then problem (1.1) admits at most one solution $u \in L_{\psi}^{p}\left(S_{T}\right)$, provided that one of the next condition holds:

$$
\begin{gathered}
0<\beta \leq N-2 s, \alpha \in \mathbb{R} ; \\
N-2 s<\beta<N, \alpha \leq 2 s ; \\
\beta=N, \alpha<2 s ; \\
\beta>N, \alpha<2 s, \alpha+\beta<2 s+N ;
\end{gathered}
$$

here $L_{\psi}^{p}\left(S_{T}\right):=\left\{f: S_{T} \rightarrow \mathbb{R}\right.$ measurable such that $\left.\int_{S_{T}}|f(x, t)|^{p} \psi(x) d x d t<\infty\right\}$. As a consequence, if $\alpha<2 s$, we have uniqueness of solutions in the class of solutions that satisfy

$$
|u(x, t)| \leq \bar{C}\left(1+|x|^{2}\right)^{\frac{\sigma}{2}} \text { for all } x \in \mathbb{R}^{N}, t>0,
$$

for some $\bar{C}>0$ and $\sigma \in(0,2 s-\alpha)$.

In the present paper, where we use completely different methods from those in 25, we always assume that

$\left(H_{0}\right) \quad$ there exist $C_{0}>0, \alpha>2 s$ such that $a(x) \geq C_{0}\left(1+|x|^{2}\right)^{\frac{\alpha}{2}}$ for all $x \in \mathbb{R}^{N}$.

Clearly, this case is not covered by [1, [19, 20], since (1.5) is not satisfied. Moreover, hypothesis $\left(H_{0}\right)$ excludes that (1.7), (1.8), (1.9) can hold; in the sequel we also discuss the case in which both $\left(H_{0}\right)$ and (1.6) hold. 
It is worth mentioning that the unbounded diffusion coefficient $a(x)$ is very important for the applications, see for instance, for the local case, 2 , 8], 11, 22, 24. Clearly, the same models with the unbounded diffusion coefficient $a(x)$ occurs when considering nonlocal diffusion, for instance, in association with non-Gaussian stochastic processes, that, starting from any point in $\mathbb{R}^{N}$, can reach infinity (see, e.g., [5]).

We prove (see Theorem 2.7) that there exists a unique solution of problem (1.1) such that (1.4) is satisfied, provided (1.3) holds; furthermore,

$$
|u| \leq C e^{\beta T} \text { in } \mathbb{R}^{N} \times[0, T],
$$

for some $C>0$ and $\beta>0$. This result generalizes to the case of nonlocal operator the results in 12 and in 18 .

In proving this result, at first for any $j \in \mathbb{N}$, we consider the viscosity solution of a suitable approximate problem in a large cylinder $B_{j} \times(0, T]$; here and hereafter for each $R>0, B_{R}:=\left\{x \in \mathbb{R}^{N}:|x|<R\right\}$. For such problem existence, uniqueness and regularity results have been given in [3], 4]. Then using suitable super- and subsolutions and standard compactness arguments we obtain the existence of a solution of problem (1.1), satifying the estimate (1.10), which depends on $T$. Then, in order to show that condition (1.4) holds, proper sub- and supersolutions are introduced (see (4.25) and (4.38) below). In the construction of these sub- and supersolutions, which also depend on the time variable $t$, a special role is played by a supersolution $V \in C^{2}\left(\mathbb{R}^{N}\right)$ of equation

$$
-a(-\Delta)^{s} V=-1 \quad \text { in } \mathbb{R}^{N} \backslash \bar{B}_{R_{0}}
$$

for some $R_{0}>0$, such that

$$
V(x)>0 \quad \text { for all } x \in \mathbb{R}^{N}, \quad \lim _{|x| \rightarrow \infty} V(x)=0,
$$

which has been appropriately constructed (see Proposition 3.1).

Moreover, we show that similar results hold for problem

$$
\begin{cases}\partial_{t} u=-a(-\Delta)^{s} u+c u+f & \text { in } \mathbb{R}^{N} \times(0, \infty) \\ u=u_{0} & \text { in } \mathbb{R}^{N} \times\{0\},\end{cases}
$$

provided $c \leq 0$ (see Theorem 2.8). Note that, in this case, condition (1.4) is replaced by

$$
\lim _{|x| \rightarrow \infty} u(x, t)=g(t) \quad \text { uniformly with respect to } t \in[0, \infty) .
$$

In order to impose condition (1.14), we need to show preliminarily that the solution satisfies the bound

$$
|u| \leq C \text { in } \mathbb{R}^{N} \times(0, \infty),
$$

which is global in time. In order to obtain this estimate, we use a positive viscosity supersolution $h \in C\left(\mathbb{R}^{N}\right)$ of equation

$$
-a(-\Delta)^{s} h=-1 \quad \text { in } \mathbb{R}^{N} .
$$

Note that the proof of the existence of such a supersolution $h$ is rather technical (see Proposition [3.2); indeed, we also show that

$$
h(x)>0 \quad \text { for all } x \in \mathbb{R}^{N}, \quad \lim _{|x| \rightarrow \infty} h(x)=0 .
$$

Let us describe in general terms the deep relation between our results and stochastic calculus for jump processes. In fact, equation (1.16) completed with condition (1.17) can be 
regarded as the counterpart on $\mathbb{R}^{N}$ for the operator $a(-\Delta)^{s}$ of the first exit-time problem in a bounded domain for $(-\Delta)^{s}$. Note that the first exit-time problem in $B_{R}$, in the case $a \equiv 1$, has been studied in [6], [10. In fact, in [6] and in [10] it is outlined the connection between the so-called first exit time problem

$$
\begin{cases}-(-\Delta)^{s} u=-1 & \text { in } B_{R} \\ u=0 & \text { in }\left(\mathbb{R}^{N} \backslash B_{R}\right),\end{cases}
$$

and the first exit-time from $B_{R}$ of the jump process associated to $(-\Delta)^{s}$, starting from any point in $B_{R}$. Moreover, it is well-known that if any point of the boundary of a bounded domain of $\mathbb{R}^{N}$ can be reached by the jump process associated to a nonlocal diffusion operator starting from points inside the domain, then the Dirichlet problem admits a unique solution that takes continuously a given datum at the boundary (see, e.g., [27]).

Now, equation (1.16), completed with condition (1.17), corresponds to problem (1.18) in the limit case $R=\infty$, and it is somehow related to reachability of infinity by the jump process associated to the operator $a(-\Delta)^{s}$ (see [5], [11]). In particular, from the existence of the supersolution $h$ it follows that infinity can actually be attained by the jump process starting from any point $x_{0} \in \mathbb{R}^{N}$. This property is usually expressed saying that the process is transient. Therefore one can expect that there exists a unique solution of problem (1.1) which satisfies conditions of Dirichlet type at infinity. Indeed, we prove this.

We should mention that, to the best of our knowledge, in the literature no results concerning the prescription of general Dirichlet conditions at infinity for solutions of nonlocal parabolic (or elliptic) equations have been obtained before the present paper .

Finally, we prove that the solution $u(x, t)$ of problem (1.13) satisfying (1.14) admits a limit function as $t \rightarrow \infty$. In fact, the function

$$
W(x):=\lim _{t \rightarrow \infty} u(x, t) \quad\left(x \in \mathbb{R}^{N}\right)
$$

is the unique solution of equation (1.2) such that

$$
\lim _{|x| \rightarrow \infty} W(x)=\gamma
$$

provided

$$
\gamma=\lim _{t \rightarrow \infty} g(t)
$$

(see Theorem 2.11). Such result is shown by adapting to the present situation the method of sub- and supersolutions used in [26] in the case of bounded domains of $\mathbb{R}^{N}$ for "local" parabolic equations. Indeed, some important changes are in order, in view of the nonlocal character of the problem and since we prescribe conditions as $|x| \rightarrow \infty$.

Now, let us discuss the case that both $\left(H_{0}\right)$ and (1.6) hold. In view of existence results described above, for any $g_{1}, g_{2} \in C([0, T])$ with $g_{1} \not \equiv g_{2}$ there exist two solutions $u_{1}$ and $u_{2}$ of problem (1.1) such that

$$
u_{1}(x, t) \rightarrow g_{1}(t), \quad u_{2}(x, t) \rightarrow g_{2}(t) \quad \text { as }|x| \rightarrow \infty, \quad \text { uniformly for } t \in[0, T] .
$$

Set $w:=u_{1}-u_{2}$. Since $g_{1} \not \equiv g_{2}$, there exists $t_{0} \in[0, T]$ such that

$$
w\left(x, t_{0}\right) \rightarrow g_{1}\left(t_{0}\right)-g_{2}\left(t_{0}\right) \neq 0 \quad \text { as }|x| \rightarrow \infty .
$$

Therefore, $w \notin L_{\psi}^{p}\left(S_{T}\right)$, with the choice of $\beta$ required in (1.6). Hence, the uniqueness result in [25] cannot be applied to conclude that $w \equiv 0$. So, obviously, the results in [25] and those described above are not in contradiction. 
(b) Ellipitc equations. In the local case, some existence and uniqueness results for equations (1.2) with $s=1$ can be deduced from general results in 24]. Moreover, the case $0<s<1$ has been treated in [25]. In particular, it is shown that uniqueness results in $L_{\psi}^{p}\left(\mathbb{R}^{N}\right)$, analogous to those recalled above for the parabolic problem, holds, if $c \leq-c_{0}$ with $c_{0}>0$ large enough . Consequently, if $\alpha<2 s$, we have uniqueness of solutions in the class of solutions that satisfy

$$
|u(x)| \leq \bar{C}\left(1+|x|^{2}\right)^{\frac{\sigma}{2}} \quad \text { for all } x \in \mathbb{R}^{N},
$$

for some $\bar{C}>0$ and $\sigma \in(0,2 s-\alpha)$. On the other hand, only requiring that $c_{0}>0$, it is shown uniqueness in $L_{(1+|x|)^{N-2 s+\alpha}}^{p}\left(\mathbb{R}^{N}\right)$, if $\alpha<2 s$.

From the result concerning the asymptotic behaviour of solutions of problem (1.13) recalled in $(a)$ above, we can infer that for any $\gamma \in \mathbb{R}$ there exists a unique solution $u$ of equation (1.2), which satisfies

$$
\lim _{|x| \rightarrow \infty} u(x)=\gamma
$$

However, we also prove this existence and uniqueness result independently, without using results for parabolic problems. In fact, we solve a proper approximate problem in a large ball $B_{j}$ for any $j \in \mathbb{N}$. In order to obtain a uniform bound, for any $j \in \mathbb{N}$, for the solutions of such problems we use in crucial way the supersolution $h$ of equation (1.16). Then, by standard compactness tools, we get a solution of equation (1.2). Using again the supersolution $h$, and in particular the fact that (1.17) holds, we impose that (1.21) holds.

We devote the forthcoming Section 2 to the precise statement of the main results obtained in this paper (see in particular Subsection 2.1).

\section{Mathematical framework and results}

The fractional Laplacian $(-\Delta)^{s}$ can be defined by Fourier transform. Namely, for any function $g$ in the Schwartz class $\mathcal{S}$, we say that

$$
(-\Delta)^{\sigma / 2} g=h
$$

if

$$
\hat{h}(\xi)=|\xi|^{\sigma} \hat{g}(\xi)
$$

Here, we used the notation $\hat{h}=\mathfrak{F} h$ for the Fourier transform of $h$. Furthermore, consider the space

$$
\mathcal{L}^{s}\left(\mathbb{R}^{N}\right):=\left\{u: \mathbb{R}^{N} \rightarrow \mathbb{R} \text { measurable } \mid \int_{\mathbb{R}^{N}} \frac{|u(x)|}{1+|x|^{n+2 s}} d x<\infty\right\},
$$

endowed with the norm

$$
\|u\|_{\mathcal{L}^{s}\left(\mathbb{R}^{N}\right)}:=\int_{\mathbb{R}^{N}} \frac{|u(x)|}{1+|x|^{N+2 s}} d x .
$$

If $u \in \mathcal{L}^{s}\left(\mathbb{R}^{N}\right)$ (see [27]), then $(-\Delta)^{s} u$ can be defined as a distribution, i.e., for any $\varphi \in \mathcal{S}$,

$$
\int_{\mathbb{R}^{N}} \varphi(-\Delta)^{s} u d x=\int_{\mathbb{R}^{N}} u(-\Delta)^{s} \varphi d x
$$

In addition, suppose that, for some $\gamma>0, u \in \mathcal{L}^{s}\left(\mathbb{R}^{N}\right) \cap C^{2 s+\gamma}\left(\mathbb{R}^{N}\right)$ if $s<\frac{1}{2}$, or $u \in$ $\mathcal{L}^{s}\left(\mathbb{R}^{N}\right) \cap C_{\text {loc }}^{1,2 s+\gamma-1}\left(\mathbb{R}^{N}\right)$ if $s \geq \frac{1}{2}$. Then we have

$$
(-\Delta)^{s} u(x)=C_{N, s} \text { P.V. } \int_{\mathbb{R}^{N}} \frac{u(x)-u(y)}{|x-y|^{N+2 s}} d y \quad\left(x \in \mathbb{R}^{N}\right),
$$

where

$$
C_{N, s}=\frac{2^{2 s} s \Gamma((N+2 s) / 2)}{\pi^{N / 2} \Gamma(1-s)},
$$


$\Gamma$ being the Gamma function; moreover, $(-\Delta)^{s} u \in C\left(\mathbb{R}^{N}\right)$. In the sequel, for simplicity, we shall write

$$
\int_{\mathbb{R}^{N}} \frac{u(x)-u(y)}{|x-y|^{N+2 s}} d y \equiv \text { P.V. } \int_{\mathbb{R}^{N}} \frac{u(x)-u(y)}{|x-y|^{N+2 s}} d y \quad\left(x \in \mathbb{R}^{N}\right) .
$$

Note that the constant $C_{N, s}$ satisfies the identity

$$
(-\Delta)^{s} u=\mathfrak{F}^{-1}\left(|\xi|^{2 s} \mathfrak{F} u\right), \quad \xi \in \mathbb{R}^{N}, u \in \mathcal{S},
$$

so (see [7])

$$
C_{N, s}=\left(\int_{\mathbb{R}^{N}} \frac{1-\cos \left(\xi_{1}\right)}{|\xi|^{N+2 s}} d \xi\right)^{-1}
$$

Concerning the coefficients $a$ and $c$, and the function $f$ we always make the following assumption:

$$
\left\{\begin{array}{l}
\text { (i) } \quad a \in C_{\text {loc }}^{0, \sigma}\left(\mathbb{R}^{N}\right)(\sigma \in(0,1)), \quad a(x)>0 \quad \text { for all } x \in \mathbb{R}^{N} \\
(\text { ii }) \quad c, f \in C_{l o c}^{0, \sigma}\left(\mathbb{R}^{N}\right) \cap L^{\infty}\left(\mathbb{R}^{N}\right)
\end{array}\right.
$$

Now we can give the definition of solution. Let $\Omega \subseteq \mathbb{R}^{N}$ be an open subset.

Definition 2.1 We say that a function $u$ is a subsolution to equation

$$
\partial_{t} u=-a(-\Delta)^{s} u+c u+f \quad \text { in } Q_{T}:=\Omega \times(0, T],
$$

if

(i) $u$ is upper semicontinuous in $S_{T}$;

(ii) for any open bounded subset $U \subset Q_{T}$, for any $\left(x_{0}, t_{0}\right) \in U$, for any test function $\varphi \in$ $C^{2}\left(S_{T}\right)$ such that $u\left(x_{0}, t_{0}\right)-\varphi\left(x_{0}, t_{0}\right) \geq u(x, t)-\varphi(x, t)$ for all $(x, t) \in U$, one has

$$
\partial_{t} \psi\left(x_{0}, t_{0}\right) \leq-a\left(x_{0}\right)(-\Delta)^{s} \psi\left(x_{0}, t_{0}\right)+c\left(x_{0}\right) u\left(x_{0}, t_{0}\right)+f\left(x_{0}\right),
$$

where

$$
\psi:=\left\{\begin{array}{l}
\varphi \text { in } U \\
u \text { in } S_{T} \backslash U .
\end{array}\right.
$$

Furthermore, we say that a function $u$ is a supersolution to equation (2.3) if

(i) $u$ is lower semicontinuous in $S_{T}$;

(ii) for any open bounded subset $U \subset Q_{T}$, for any $\left(x_{0}, t_{0}\right) \in U$, for any test function $\varphi \in$ $C^{2}\left(S_{T}\right)$ such that $u\left(x_{0}, t_{0}\right)-\varphi\left(x_{0}, t_{0}\right) \leq u(x, t)-\varphi(x, t)$ for all $(x, t) \in U$, one has

$$
\partial_{t} \psi\left(x_{0}, t_{0}\right) \geq-a\left(x_{0}\right)(-\Delta)^{s} \psi\left(x_{0}, t_{0}\right)+c\left(x_{0}\right) u\left(x_{0}, t_{0}\right)+f\left(x_{0}\right),
$$

where $\psi$ is defined by (2.4). Finally, we say that $u$ is a solution to equation (1.2) if it is both a subsolution and a supersolution to equation (2.3).

Let $g \in C([0, T]), u_{0} \in C\left(\mathbb{R}^{N}\right)$ with

$$
u_{0}(x, 0)=g(0) \text { for all } x \in \mathbb{R}^{N} \backslash \Omega .
$$

Consider the problem

$$
\begin{cases}\partial_{t} u=-a(-\Delta)^{s} u+c u+f & \text { in } Q_{T} \\ u=g & \text { in }\left(\mathbb{R}^{N} \backslash \Omega\right) \times(0, T] \\ u=u_{0} & \text { in } \mathbb{R}^{N} \times\{0\} .\end{cases}
$$


Definition 2.2 We say that a function $u$ is a subsolution to problem (2.6) if

(i) $u$ is upper semicontinuous in $\overline{S_{T}}$;

(ii) $u$ is a subsolution to equation (2.3);

(iii) $u(x, t) \leq g(t)$ for all $x \in \mathbb{R}^{N} \backslash \Omega, t \in(0, T]$ and $u(x, 0) \leq u_{0}(x)$ for all $x \in \mathbb{R}^{N}$.

Similarly, supersolutions are defined. Finally, we say that $u$ is a solution to problem (2.6) if it is both a subsolution and a supersolution to problem (2.6).

Observe that according to our definition, any solution of problem (2.6) takes continuously the initial datum $u_{0}$ and the boundary datum $g$.

Definition 2.3 We say that a function $u$ is a subsolution to equation

$$
a(-\Delta)^{s} u-c u=f \quad \text { in } \Omega,
$$

if

(i) $u$ is upper semicontinuous in $\mathbb{R}^{N}$;

(ii) for any open bounded subset $U \subset \Omega$, for any $x_{0} \in U$, for any test function $\varphi \in C^{2}\left(\mathbb{R}^{N}\right)$ such that $u\left(x_{0}\right)-\varphi\left(x_{0}\right) \geq u(x)-\varphi(x)$ for all $x \in U$, one has

$$
a\left(x_{0}\right)(-\Delta)^{s} \psi\left(x_{0}\right)-c\left(x_{0}\right) u\left(x_{0}\right) \leq f\left(x_{0}\right),
$$

where $\psi$ is defined by

$$
\psi:=\left\{\begin{array}{l}
\varphi \text { in } U \\
u \text { in } \mathbb{R}^{N} \backslash U .
\end{array}\right.
$$

Furthermore, we say that a function $u$ is a supersolution to equation (2.7) if

(i) $u$ is lower semicontinuous in $\mathbb{R}^{N}$;

(ii) for any open subset $U \in \Omega$, for any $x_{0} \in U$, for any test function $\varphi \in C^{2}(\Omega)$ such that $u\left(x_{0}\right)-\varphi\left(x_{0}\right) \leq u(x)-\varphi(x)$ for all $x \in U$, one has one has

$$
a\left(x_{0}\right)(-\Delta)^{s} \psi\left(x_{0}\right)-c\left(x_{0}\right) u\left(x_{0}\right) \geq f\left(x_{0}\right) .
$$

Finally, we say that $u$ is a solution to equation (2.7) if it is both a subsolution and a supersolution to equation (2.7).

Consider the following problem

$$
\begin{cases}-a(-\Delta)^{s} u-c u=f & \text { in } \Omega \\ u=\gamma & \text { in }\left(\mathbb{R}^{N} \backslash \Omega\right),\end{cases}
$$

where $\gamma \in \mathbb{R}$.

Definition 2.4 We say that a function $u$ is a subsolution to problem (2.9) if

(i) $u$ is upper semicontinuous in $\mathbb{R}^{N}$;

(ii) $u$ is a subsolution to equation (2.7);

(iii) $u(x) \leq \gamma$ for all $x \in \mathbb{R}^{N} \backslash \Omega$.

Similarly, supersolutions and solutions are defined.

In the next two Remarks we summarize existence, uniqueness and regularity results shown in [3, 4, for problems (2.6) and (2.9), that will be used in the sequel. 
Remark 2.5 Let $\Omega \subset \mathbb{R}^{N}$ be an open bounded subset with $\partial \Omega$ of class $C^{1}$. Let assumption $\left(H_{1}\right)$ be satisfied. Let $g \in C([0, T]), u_{0} \in C\left(\mathbb{R}^{N}\right) \cap L^{\infty}\left(\mathbb{R}^{N}\right)$; suppose that condition (2.5) is satisfied. We have that

(i) there exists a unique solution to problem (2.6);

(ii) the comparison principle holds for problem (2.6);

(iii) if $u$ is a solution of equation (2.3), then, for some $0<\mu<1$, for any open subset $\Omega^{\prime} \subset \subset \Omega, \tau \in(0, T]$ we have

$$
\left|u\left(x, t_{1}\right)-u\left(y, t_{2}\right)\right| \leq C\left(|x-y|^{\mu}+\left|t_{1}-t_{2}\right|^{\frac{\mu}{2 s}}\right) \quad \text { for all } x, y \in \Omega^{\prime}, t_{1}, t_{2} \in[\tau, T],
$$

for some constant $C>0$, which only depends on $\|u\|_{\infty}, N, a, c, f$.

Note that (i) - (ii) follow from [3, Section 4.3], while (iii) is a consequence of the results in [4] (see also the comments at the end of page 2 in [4]).

Remark 2.6 Let $\Omega \subset \mathbb{R}^{N}$ an open bounded subset with $\partial \Omega$ of class $C^{1}$; let $\gamma \in \mathbb{R}$. Let assumption $\left(H_{1}\right)$ be satisfied. Assume that $c \leq 0$ in $\Omega$. We have that

(i) there exists a unique solution to problem (2.9);

(ii) the comparison principle holds for problem (2.9);

(iii) if $u$ is a solution of equation (2.7), then, for some $\mu \in(0,1)$, for any open subset $\Omega^{\prime} \subset \subset \Omega$,

$$
\|u\|_{C^{0, \mu}\left(\Omega^{\prime}\right)} \leq C,
$$

for some constant $C>0$, which only depends on $\|u\|_{\infty}, N, a, c, f$.

Note that (i), (ii) follow from [3, Theorem 2]) and [4, Theorem1]), whereas from Theorem 2 and the comments at the end of page 2 in [4] it follows (iii).

\subsection{Main results: existence, uniqueness and asymptotic behaviour of solutions}

Concerning existence and uniqueness of solutions of problem (1.1) we have the next result.

Theorem 2.7 Let assumptions $\left(H_{0}\right),\left(H_{1}\right)$ be satisfied. Let $T>0$. Let $g \in C([0, T]), u_{0} \in$ $C\left(\mathbb{R}^{N}\right) \cap L^{\infty}\left(\mathbb{R}^{N}\right)$; suppose that condition (1.3) is satisfied. Then there exists a unique solution $u$ to problem (1.1) such that condition (1.4) is satisfied. Furthermore, (1.10) holds .

Under the extra hypothesis that $c \leq 0$, we have the next existence and uniqueness for problem (1.13) .

Theorem 2.8 Let assumptions $\left(H_{0}\right),\left(H_{1}\right)$ be satisfied. Let $g \in C([0, \infty)) \cap L^{\infty}((0, \infty)), u_{0} \in$ $C\left(\mathbb{R}^{N}\right) \cap L^{\infty}\left(\mathbb{R}^{N}\right), c \leq 0$; suppose that condition (1.3) is satisfied. Then there exists a unique solution to problem (1.13) such that condition (1.14) is satisfied. Furthermore, for some $C>0$, (1.15) holds .

Remark 2.9 Observe that the estimate in (1.10) depends on $T>0$, while that in (1.15) is independent of $T$. In order to get (1.15) we use the further hypothesis $c \leq 0$.

Concerning the elliptic equation (1.2) we show the next result.

Theorem 2.10 Let assumptions $\left(H_{0}\right),\left(H_{1}\right)$ be satisfied. Let $\gamma \in \mathbb{R}$; suppose that $c \leq 0$ in $\mathbb{R}^{N}$. Then there exists a unique solution to equation (1.2) such that condition (1.21) is satisfied.

The next theorem is concerned with the asymptotic behaviour as $t \rightarrow \infty$ of solutions of problem (1.13) . 
Theorem 2.11 Let assumptions of Theorem 2.8 be satisfied. Let $\gamma:=\lim _{t \rightarrow \infty} g(t)$. Let $u$ be the unique solution to problem (1.1) such that (1.4) is satisfied. Suppose that condition (1.20) holds. Then

$$
\lim _{t \rightarrow \infty} u(x, t)=W(x) \quad \text { for all } x \in \mathbb{R}^{N},
$$

where $W$ is the unique solution of equation (1.2) satisfying condition (1.19).

Remark 2.12 Note that the existence result in Theorem 2.10 can be regarded as a consequence of Theorem 2.11. In fact, from Theorem 2.11 in particular we obtain the existence of a solution $W(x):=\lim _{t \rightarrow \infty} u(x, t)$ of problem (1.2), where $u(x, t)$ is the solution of problem (1.1) with $g(t) \equiv \gamma$ and $u_{0}$ satisfying (1.3). However, in Section 4 we give an independent proof of Theorem 2.11, without using results concerning the parabolic problem. Finally, observe that the supersolution $h(x)$ of equation (1.16) plays a crucial role both in the Proof of Theorem 2.10 and in that of Theorem 2.11.

\section{Construction of stationary supersolutions}

Let us introduce the hypergeometric function

$$
{ }_{2} F_{1}(a, b, c, \sigma) \equiv F(a, b, c, \sigma)
$$

with $a, b \in \mathbb{R}, c>0, \sigma \in \mathbb{R} \backslash\{1\}$. The next limits holds (see [21, Chapters 15.2, 15.4]):

$$
\lim _{\sigma \rightarrow 1^{-}} F(a, b, c, \sigma)=\frac{\Gamma(c) \Gamma(c-a-b)}{\Gamma(c-a) \Gamma(c-b)},
$$

where $\Gamma$ is the Gamma function. Note that

$$
\Gamma(t)>0 \text { for all } t>0 .
$$

For any $C>0, \beta>0$ define the function

$$
V(x):=C\left(1+|x|^{2}\right)^{-\frac{\beta}{2}} \quad\left(x \in \mathbb{R}^{N}\right) .
$$

Concerning the function $V$, we show the next result.

Proposition 3.1 Let assumptions $\left(H_{0}\right),\left(H_{1}\right)-(i)$ be satisfied. There exists $C>0, \beta>0$ and $R_{0}>0$ such that the function $V$ satisfies

$$
-a(x)(-\Delta)^{s} V(x) \leq-1 \quad \text { for all } x \in \mathbb{R}^{N} \backslash \bar{B}_{R_{0}} .
$$

In particular, $V$ is a supersolution of equation (1.11) in the sense of Definition 2.3. Moreover, (1.12) holds .

Proof. To begin with, observe that since $V \in C^{\infty}\left(\mathbb{R}^{N}\right)$, we have that $(-\Delta)^{s} V \in C\left(\mathbb{R}^{N}\right)$ (see Section 22). From the proof of Corollary 4.1 in [9] it follows that, for some constant $\check{C}>0$,

$$
-(-\Delta)^{s} V(x)=-C \check{C} F\left(a, b, c,-|x|^{2}\right) \quad \text { whenever }|x|>1,
$$

with

$$
a=\frac{N}{2}+s, \quad b=\frac{\beta}{2}+s, \quad c=\frac{N}{2} .
$$

By Pfaff's transformation,

$$
F\left(a, b, c,-|x|^{2}\right)=\frac{1}{\left(1+|x|^{2}\right)^{b}} F\left(c-a, b, c, \frac{|x|^{2}}{1+|x|^{2}}\right) \quad \text { for all } x \in \mathbb{R}^{N} \backslash B_{1} .
$$


Suppose that $0<\beta<N$. Hence, as a consequence of (3.10) we get

$$
\lim _{|x| \rightarrow \infty} F\left(-s, \frac{\beta}{2}+s, \frac{N}{2}, \frac{|x|^{2}}{1+|x|^{2}}\right)=\frac{\Gamma\left(\frac{N}{2}\right) \Gamma\left(\frac{N-\beta}{2}\right)}{\Gamma\left(\frac{N}{2}+s\right) \Gamma\left(\frac{N-\beta}{2}-s\right)}=: K .
$$

Now, we choose $0<\beta<N-2 s$, so we have $K>0$. Due to (3.14), (3.15), (3.16), we can find $R_{0}>0$ such that

$$
-(-\Delta)^{s} V(x) \leq-\frac{C \check{C} K}{2\left(1+|x|^{2}\right)^{\frac{\beta}{2}+s}} \quad \text { for all } x \in \mathbb{R}^{N} \backslash B_{R_{0}} .
$$

If we select $\beta$ that also satisfies $0<\beta \leq \alpha-2 s$, then (3.17) and $\left(H_{0}\right)$ yields (3.13), provided that

$$
C \geq \frac{2}{C_{0} \check{C} K}
$$

Since $V \in C^{\infty}\left(\mathbb{R}^{N}\right)$, it easily follows that $V$ is a supersolution of equation (3.13) in the sense of Definition 2.3. Finally, the properties in (1.12) immediately follow from the very definition of $V$.

Proposition 3.2 Let assumptions $\left(H_{0}\right),\left(H_{1}\right)-(i)$ be satisfied. Then there exists a supersolution $h$ of equation (1.16) in the sense of Definition 2.3, which satisfies (1.17).

Proof. Let $R_{0}, C$ and $V$ be given by Proposition 3.1. Take $\hat{R}>R_{0}$. From the results in 10 . it follows that, for a certain $C_{1}=C_{1}(N, s)>0$, the function

$$
\hat{W}(x) \equiv \hat{W}(|x|):=C_{1}\left(\hat{R}^{2}-|x|^{2}\right)_{+}^{s / 2} \quad\left(x \in \mathbb{R}^{N}\right) .
$$

solves

$$
\begin{cases}-(-\Delta)^{s} u=-1 & \text { in } B_{\hat{R}} \\ u=0 & \text { in } \mathbb{R}^{N} \backslash B_{\hat{R}} .\end{cases}
$$

Hence, it easily follows that for each $\mu_{0}>0, \mu_{1} \geq \mu_{0} \max _{\bar{B}_{\hat{R}}} \frac{1}{a}$ and $\mu_{2}>0$, the function

$$
W(x) \equiv W(|x|):=\mu_{1} \hat{W}(x)+\mu_{2} \quad\left(x \in \mathbb{R}^{N}\right)
$$

is a supersolution of problem

$$
\begin{cases}-a(x)(-\Delta)^{s} u=-\mu_{0} & \text { in } B_{\hat{R}} \\ u=\mu_{2} & \text { in } \mathbb{R}^{N} \backslash B_{\hat{R}} .\end{cases}
$$

For any $\tilde{C}>0$ set

$$
\tilde{V}(x) \equiv \tilde{V}(|x|):=\tilde{C} V(|x|) \quad\left(x \in \mathbb{R}^{N}\right) .
$$

We see that for suitable $\mu_{2}>0, \hat{R}>0, \tilde{C}>0$, possibly depending on $C, C_{1}, \mu_{1}, \beta, s$, we have

$$
\tilde{V}(0)>W(0), \quad \tilde{V}<W \quad \text { in }\left[\frac{\hat{R}}{2}, \hat{R}\right] .
$$

In fact, if

$$
\tilde{C} C\left[1+\left(\frac{\hat{R}}{2}\right)^{2}\right]^{-\frac{\beta}{2}}<\mu_{2}<\tilde{C} C-\mu_{1} C_{1} \hat{R}^{s}
$$


then (3.20) holds. Now, if we take

$$
\tilde{C}=\hat{R}^{s+\nu}, \quad \mu_{2}=\hat{R}^{s+\nu-\beta+\delta}
$$

with

$$
0<\delta<\beta, \quad \nu>\max \{0, \beta-s+2\},
$$

then, it is direct to see that, for $\hat{R}>0$ large enough, (3.21), and so (3.20), holds.

In view of (3.20), there exists $\bar{R} \in(0, \hat{R} / 2)$ such that $W(\bar{R})=\tilde{V}(\bar{R})$. Indeed, such $\bar{R}$ is unique. To see this, take any $\bar{R}>0$ such that $W(\bar{R})=\tilde{V}(\bar{R})$. In view of (3.20) and the very definition of $W$ and $\tilde{V}$ we have that $\bar{R} \in(0, \hat{R} / 2)$. So,

$$
\hat{R}^{2}-\bar{R}^{2} \geq 1,
$$

provided $\hat{R}>2$. Moreover, it is direct to check that if we show that

$$
W^{\prime}(\bar{R})>\tilde{V}^{\prime}(\bar{R}),
$$

then such $\bar{R}$ is unique. In order to show (3.24), note that (3.24) is equivalent to

$$
s \mu_{1} C_{1}\left(1+\bar{R}^{2}\right)<\beta\left(\hat{R}^{2}-\bar{R}^{2}\right)^{1-\frac{s}{2}} \tilde{V}(\bar{R}) .
$$

Now, since

$$
s \mu_{1} C_{1}\left(1+\bar{R}^{2}\right) \leq s \mu_{1} C_{1}\left(1+\hat{R}^{2}\right),
$$

in view of (3.23), (3.25) follows if we prove that

$$
s \mu_{1} C_{1}\left(1+\hat{R}^{2}\right) \leq \beta \mu_{2} .
$$

Clearly, (3.26) is a direct consequence of (3.22), provided that $\hat{R}>0$ is large enough. Hence, we have that (3.24) is satisfied, and so $\bar{R}$ is unique. Therefore,

$$
\tilde{V} \geq W \quad \text { in } B_{\bar{R}}, \quad \tilde{V}(\bar{R})=W(\bar{R}), \quad \tilde{V} \leq W \quad \text { in } \mathbb{R}^{N} \backslash B_{\bar{R}} .
$$

Furthermore, since

$$
\tilde{V}(\bar{R})=W(\bar{R})
$$

we get

$$
\mu_{2}(1+\bar{R})^{\frac{\beta}{2}} \geq \tilde{C} C-\mu_{1} C_{1} \hat{R}^{s}\left(1+\bar{R}^{2}\right)^{\frac{\beta}{2}}
$$

thus, (3.22) yields

$$
\left(1+\bar{R}^{2}\right)^{\frac{\beta}{2}} \geq \frac{C \hat{R}^{s+\nu}}{\hat{R}^{s+\nu+\delta-\beta}+\mu_{1} C_{1} \hat{R}^{s}} .
$$

This implies that we can choose $\hat{R}>0$ so large that $\bar{R} \in\left(R_{0}, \hat{R} / 2\right)$.

Define

$$
h:=\min \{\tilde{V}, W\} \quad \text { in } \mathbb{R}^{N} .
$$

We claim that $h$ is a supersolution of equation

$$
-a(-\Delta)^{s} h=-\min \left\{\mu_{0}, \tilde{C}\right\} \quad \text { in } \mathbb{R}^{N} .
$$

In fact, since $V$ is a supersolution of equation (1.11), by Definition 2.3 and (2.2), for any open bounded subset $\Omega^{\prime} \subset \mathbb{R}^{N} \backslash \bar{B}_{R_{0}}$, for any $x_{0} \in \Omega^{\prime}$, for any test function $\varphi \in C^{2}\left(\mathbb{R}^{N}\right)$ such that $\tilde{V}\left(x_{0}\right)-\varphi\left(x_{0}\right) \leq \tilde{V}(x)-\varphi(x)$ for all $x \in \Omega^{\prime}$, one has

$$
a\left(x_{0}\right) C_{N, s} \int_{\mathbb{R}^{N}} \frac{\psi\left(x_{0}\right)-\psi(y)}{\left|x_{0}-y\right|^{N+2 s}} d y \geq \tilde{C}
$$


where $\psi$ is defined by (2.8) with $u$ replaced by $\tilde{V}$ and $U$ by $\Omega^{\prime}$. Hence

$$
a\left(x_{0}\right) C_{N, s}\left\{\int_{\Omega^{\prime}} \frac{\varphi\left(x_{0}\right)-\varphi(y)}{\left|x_{0}-y\right|^{N+2 s}} d y+\int_{\mathbb{R}^{N} \backslash \Omega^{\prime}} \frac{\tilde{V}\left(x_{0}\right)-\tilde{V}(y)}{\left|x_{0}-y\right|^{N+2 s}} d y\right\} \geq \tilde{C} .
$$

Similarly, since $W$ is a supersolution of problem (3.19), we have that for any open bounded subset $U \subset B_{R_{0}}$, for any $x_{0} \in U$, for any test function $\varphi \in C^{2}\left(\mathbb{R}^{N}\right)$ such that $W\left(x_{0}\right)-\varphi\left(x_{0}\right) \leq$ $W(x)-\varphi(x)$ for all $x \in U$, one has

$$
a\left(x_{0}\right) C_{N, s}\left\{\int_{U} \frac{\varphi\left(x_{0}\right)-\varphi(y)}{\left|x_{0}-y\right|^{N+2 s}} d y+\int_{\mathbb{R}^{N} \backslash U} \frac{W\left(x_{0}\right)-W(y)}{\left|x_{0}-y\right|^{N+2 s}} d y\right\} \geq \mu_{0} .
$$

Now, take any $x_{0} \in \mathbb{R}^{N}$ with $\left|x_{0}\right| \geq \bar{R}$, any open bounded subset $U \subset \mathbb{R}^{N}$ with $x_{0} \in U$, and any test function $\varphi \in C^{2}\left(\mathbb{R}^{N}\right)$ such that $h\left(x_{0}\right)-\varphi\left(x_{0}\right) \leq h(x)-\varphi(x)$ for all $x \in U$. Set

$$
\psi:= \begin{cases}\varphi & \text { in } U \\ h & \text { in } \mathbb{R}^{N} \backslash U .\end{cases}
$$

Note that, due to (3.27), we have

$$
h\left(x_{0}\right)=\tilde{V}\left(x_{0}\right) .
$$

For any $0<\epsilon<\bar{R}-R_{0}$, we have $\mathcal{U}_{1}:=U \cap\left(\mathbb{R}^{N} \backslash B_{R_{0}+\epsilon}\right) \subset \mathbb{R}^{N} \backslash \bar{B}_{R_{0}}, x_{0} \in \mathcal{U}_{1}$. Moreover,

$$
\varphi(x) \leq \tilde{V}(x) \text { for all } x \in \mathcal{U}_{1}, \quad \varphi\left(x_{0}\right)=\tilde{V}\left(x_{0}\right) .
$$

So, from (3.28) with $\Omega^{\prime}=\mathcal{U}_{1}$ we get

$$
a\left(x_{0}\right) C_{N, s}\left\{\int_{\mathcal{U}_{1}} \frac{\varphi\left(x_{0}\right)-\varphi(y)}{\left|x_{0}-y\right|^{N+2 s}} d y+\int_{\mathbb{R}^{N} \backslash \mathcal{U}_{1}} \frac{\tilde{V}\left(x_{0}\right)-\tilde{V}(y)}{\left|x_{0}-y\right|^{N+2 s}} d y\right\} \geq \tilde{C} .
$$

Due to (3.27) and (3.31), since $h \leq \tilde{V}$ in $\mathbb{R}^{N}$, we have

$$
a\left(x_{0}\right) C_{N, s}\left\{\int_{\mathcal{U}_{1}} \frac{\varphi\left(x_{0}\right)-\varphi(y)}{\left|x_{0}-y\right|^{N+2 s}} d y+\int_{\mathbb{R}^{N} \backslash \mathcal{U}_{1}} \frac{h\left(x_{0}\right)-h(y)}{\left|x_{0}-y\right|^{N+2 s}} d y\right\} \geq \tilde{C} .
$$

Set $\mathcal{U}_{2}:=U \cap B_{R_{0}+\epsilon}$. In view of (3.34), since $\varphi\left(x_{0}\right)-\varphi(y) \geq h\left(x_{0}\right)-h(y)$ for all $y \in \mathcal{U}_{2}$ we have

$$
\begin{array}{r}
a\left(x_{0}\right) C_{N, s} \int_{U} \frac{\psi\left(x_{0}\right)-\psi(y)}{\left|x_{0}-y\right|^{N+2 s}} d y \\
=a\left(x_{0}\right) C_{N, s}\left\{\int_{U} \frac{\varphi\left(x_{0}\right)-\varphi(y)}{\left|x_{0}-y\right|^{N+2 s}} d y+\int_{\mathbb{R}^{N} \backslash U} \frac{h\left(x_{0}\right)-h(y)}{\left|x_{0}-y\right|^{N+2 s}} d y\right\} \\
=a\left(x_{0}\right) C_{N, s}\left\{\int_{\mathcal{U}_{1}} \frac{\varphi\left(x_{0}\right)-\varphi(y)}{\left|x_{0}-y\right|^{N+2 s}} d y+\int_{\mathbb{R}^{N} \backslash \mathcal{U}_{1}} \frac{h\left(x_{0}\right)-h(y)}{\left|x_{0}-y\right|^{N+2 s}} d y\right. \\
\left.-\int_{\mathcal{U}_{2}} \frac{h\left(x_{0}\right)-h(y)}{\left|x_{0}-y\right|^{N+2 s}} d y+\int_{\mathcal{U}_{2}} \frac{\varphi\left(x_{0}\right)-\varphi(y)}{\left|x_{0}-y\right|^{N+2 s}} d y\right\} \geq \tilde{C} .
\end{array}
$$

Now, take any $x_{0} \in \mathbb{R}^{N}$ with $\left|x_{0}\right|<\bar{R}$, any open bounded subset $U \subset \mathbb{R}^{N}$ with $x_{0} \in U$, and any test function $\varphi \in C^{2}\left(\mathbb{R}^{N}\right)$ such that $h\left(x_{0}\right)-\varphi\left(x_{0}\right) \leq h(x)-\varphi(x)$ for all $x \in U$. Let $\psi$ be defined by (3.30). Note that (3.27) gives

$$
h\left(x_{0}\right)=W\left(x_{0}\right) .
$$


For any $0<\epsilon<\bar{R}-R_{0}$ we have $\mathcal{U}_{1}:=U \cap B_{\bar{R}-\epsilon} \subset \bar{B}_{\bar{R}}, x_{0} \in \mathcal{U}_{1}$. Moreover,

$$
\varphi(x) \leq W(x) \text { for all } x \in \mathcal{U}_{1}, \quad \varphi\left(x_{0}\right)=W\left(x_{0}\right) .
$$

So, from (3.29) with $\Omega^{\prime}=\mathcal{U}_{1}$ we get

$$
a\left(x_{0}\right) C_{N, s}\left\{\int_{\mathcal{U}_{1}} \frac{\varphi\left(x_{0}\right)-\varphi(y)}{\left|x_{0}-y\right|^{N+2 s}} d y+\int_{\mathbb{R}^{N} \backslash \mathcal{U}_{1}} \frac{W\left(x_{0}\right)-W(y)}{\left|x_{0}-y\right|^{N+2 s}} d y\right\} \geq \mu_{0} .
$$

Due to (3.36) and (3.38), since $h \leq W$ in $\mathbb{R}^{N}$, we have

$$
a\left(x_{0}\right) C_{N, s}\left\{\int_{\mathcal{U}_{1}} \frac{\varphi\left(x_{0}\right)-\varphi(y)}{\left|x_{0}-y\right|^{N+2 s}} d y+\int_{\mathbb{R}^{N} \backslash \mathcal{U}_{1}} \frac{h\left(x_{0}\right)-h(y)}{\left|x_{0}-y\right|^{N+2 s}} d y\right\} \geq \mu_{0} .
$$

Set $\mathcal{U}_{2}:=U \cap\left(\mathbb{R}^{N} \backslash B_{\bar{R}-\epsilon}\right)$. In view of (3.39) , since $\varphi\left(x_{0}\right)-\varphi(y) \geq h\left(x_{0}\right)-h(y)$ for all $y \in \mathcal{U}_{2}$ we have

$$
\begin{array}{r}
a\left(x_{0}\right) C_{N, s} \int_{U} \frac{\psi\left(x_{0}\right)-\psi(y)}{\left|x_{0}-y\right|^{N+2 s}} d y \\
=a\left(x_{0}\right) C_{N, s}\left\{\int_{U} \frac{\varphi\left(x_{0}\right)-\varphi(y)}{\left|x_{0}-y\right|^{N+2 s}} d y+\int_{\mathbb{R}^{N} \backslash U} \frac{h\left(x_{0}\right)-h(y)}{\left|x_{0}-y\right|^{N+2 s}} d y\right\} \\
=a\left(x_{0}\right) C_{N, s}\left\{\int_{\mathcal{U}_{1}} \frac{\varphi\left(x_{0}\right)-\varphi(y)}{\left|x_{0}-y\right|^{N+2 s}} d y+\int_{\mathbb{R}^{N} \backslash \mathcal{U}_{1}} \frac{h\left(x_{0}\right)-h(y)}{\left|x_{0}-y\right|^{N+2 s}} d y\right. \\
\left.-\int_{\mathcal{U}_{2}} \frac{h\left(x_{0}\right)-h(y)}{\left|x_{0}-y\right|^{N+2 s}} d y+\int_{\mathcal{U}_{2}} \frac{\varphi\left(x_{0}\right)-\varphi(y)}{\left|x_{0}-y\right|^{N+2 s}} d y\right\} \geq \mu_{0} .
\end{array}
$$

From (3.35) and (3.40) the claim follows. Therefore,

$$
h:=\bar{C} h \quad \text { in } \mathbb{R}^{N},
$$

with $\bar{C} \geq \max \left\{\frac{1}{\mu_{0}}, \frac{1}{C}\right\}$, is a supersolution of equation (1.16); moreover, it is immediately seen that it satisfies (1.17).

\section{Proofs of existence and uniqueness results}

To begin with, let us show the next quite standard comparison principle.

Proposition 4.1 Let assumptions $\left(H_{0}\right),\left(H_{1}\right)$ be satisfied. Let $u$ be a subsolution of problem (1.1), let $v$ be a supersolution of problem (1.1). Suppose that both

$$
\limsup _{|x| \rightarrow \infty}(u-v) \leq 0 \quad \text { uniformly for } t \in[0, T] .
$$

Then

$$
u \leq v \quad \text { in } S_{T} .
$$

Proof. Set $w:=u-v$. Let $\epsilon>0$. Then there exists $R_{\epsilon}>0$ such that

$$
|w(x, t)| \leq \epsilon \quad \text { for all } x \in \mathbb{R}^{N} \backslash B_{R_{\epsilon}}, t \in[0, T] .
$$

Hence $w$ is a subsolution of problem

$$
\begin{cases}\partial_{t} v=-a(-\Delta)^{s} v+c v & \text { in } B_{R_{\epsilon}} \times(0, T] \\ v=\epsilon & \text { in }\left(\mathbb{R}^{N} \backslash B_{R_{\epsilon}}\right) \times(0, T] \\ v=0 & \text { in } \mathbb{R}^{N} \times\{0\} .\end{cases}
$$


Moreover, it is easily seen that the function

$$
z(x, t):=\epsilon e^{\|c\|_{\infty} t} \quad\left(x \in \mathbb{R}^{N}, t \in[0, T]\right)
$$

is a supersolution of problem (4.1). By the comparison principle (see Remark 2.5),

$$
w \leq z \quad \text { in } \mathbb{R}^{N} \times[0, T] .
$$

Similarly, it can be shown that

$$
w \geq-z \quad \text { in } \mathbb{R}^{N} \times[0, T] .
$$

Letting $\epsilon \rightarrow 0^{+}$, we get $w=0$ in $\mathbb{R}^{N} \times[0, T]$. Hence the proof is complete. that

Let us prove Theorem 2.7. Hereafter, $\left\{\zeta_{j}\right\} \subset C_{c}^{\infty}\left(B_{j}\right)$ will be a sequence of functions such

$$
0 \leq \zeta_{j} \leq 1, \quad \zeta_{j} \equiv 1 \text { in } B_{j / 2} \text { for each } j \in \mathbb{N} .
$$

Proof of Theorem 2.7. For any $j \in \mathbb{N}$ let $u_{j}$ be the unique solution (see Remark 2.5) of the problem

$$
\begin{cases}\partial_{t} u=-a(-\Delta)^{s} u+c u+f & \text { in } B_{j} \times(0, T] \\ u=g & \text { in }\left(\mathbb{R}^{N} \backslash B_{j}\right) \times(0, T] \\ u=u_{0, j} & \text { in } \mathbb{R}^{N} \times\{0\},\end{cases}
$$

where

$$
u_{0, j}(x):=\zeta_{j}(x) u_{0}(x)+\left[1-\zeta_{j}(x)\right] g(0) \text { for all } x \in \bar{B}_{j} .
$$

It is easily seen that the function

$$
\bar{v}(x, t):=C e^{\beta t} \quad\left((x, t) \in \mathbb{R}^{N} \times[0, T]\right)
$$

is a supersolution of problem (4.5) for any $j \in \mathbb{N}$, provided that

$$
\beta \geq 1+\|c\|_{\infty}, \quad C \geq \max \left\{\|f\|_{\infty},\|g\|_{\infty},\left\|u_{0}\right\|_{\infty}\right\} .
$$

Thus, by the comparison principle (see Remark 2.5),

$$
u_{j}(x, t) \leq \bar{v}(x, t) \quad \text { for all }(x, t) \in \mathbb{R}^{N} \times[0, T] .
$$

Furthermore, the function

$$
\underline{v}(x, t):=-C e^{\beta t} \quad\left((x, t) \in \mathbb{R}^{N} \times[0, T]\right)
$$

is a subsolution of problem (4.5) for any $j \in \mathbb{N}$. Thus, by the comparison principle,

$$
u_{j}(x, t) \geq \underline{v}(x, t) \quad \text { for all }(x, t) \in \mathbb{R}^{N} \times[0, T] .
$$

From (4.6)-(4.7) we obtain

$$
\left|u_{j}(x, t)\right| \leq C e^{\beta T}=: K_{T} \quad \text { for all }(x, t) \in \mathbb{R}^{N} \times[0, T] .
$$

By the a priori estimates recalled in Remark 2.6 $(i i i)$ and usual compactness arguments, there exists a subsequence $\left\{u_{j_{k}}\right\} \subset\left\{u_{j}\right\}$ and a function $u \in C\left(S_{T}\right)$ such that

$$
u:=\lim _{k \rightarrow \infty} u_{j_{k}} \text { uniformly in } D \times[\tau, T],
$$


for any compact subset $D \subset \mathbb{R}^{N}$ and for any $\tau \in(0, T)$. For simplicity we still denote $\left\{u_{j_{k}}\right\}$ by $\left\{u_{j}\right\}$. In view of stability properties of viscosity solutions under local uniform convergence, the function $u$ is a solution of equation

$$
\partial_{t} u=-a(-\Delta)^{s} u+c u+f \quad \text { in } \mathbb{R}^{N} \times(0, T] .
$$

Claim 1: We have that

$$
\lim _{t \rightarrow 0^{+}} u(x, t)=u_{0}(x) \quad \text { for any } x \in \mathbb{R}^{N} .
$$

In fact, let $x_{0} \in \mathbb{R}^{N}$. Take $j_{0} \in \mathbb{N}$ so large that $x_{0} \in B_{j_{0} / 2}$. In view of the definition of $\left\{\zeta_{j}\right\}$ (see (4.4)) there exists $\delta_{0}>0$ such that for any $j \geq j_{0}$

$$
u_{j}(x, 0)=u_{0, j}(x)=u_{0}(x) \text { for all } x \in B_{\delta_{0}}\left(x_{0}\right) .
$$

Since $u_{0} \in C\left(\mathbb{R}^{N}\right)$, for any $0<\epsilon<1$ there exists $\delta \in\left(0, \delta_{0}\right)$ such that

$$
-\epsilon<u_{0}(x)-u_{0}\left(x_{0}\right)<\epsilon \text { for all } x \in B_{\delta}\left(x_{0}\right) .
$$

From (4.9), (4.10) it follows that for any $0<\epsilon<1$ and any $j \geq j_{0}$ there holds

$$
-\epsilon<u_{j}(x, 0)-u_{0}\left(x_{0}\right)<\epsilon \text { for all } x \in B_{\delta}\left(x_{0}\right) .
$$

Let

$$
\chi\left(x ; x_{0}\right) \equiv \chi(x):=\left|x-x_{0}\right|^{2} \quad\left(x \in \mathbb{R}^{N}\right) .
$$

Since $\chi \in C^{\infty}\left(\mathbb{R}^{N}\right)$, we have that $(-\Delta)^{s} \chi \in C\left(\mathbb{R}^{N}\right)$. Define

$$
\begin{gathered}
h(x, t):=[\chi(x)+A t] e^{\eta t} \quad\left(x \in \mathbb{R}^{N}, t \in[0, \delta]\right), \\
\bar{v}(x, t):=M h(x, t)+u_{0}\left(x_{0}\right)+\epsilon \quad\left(x \in \mathbb{R}^{N}, t \in[0, \delta]\right),
\end{gathered}
$$

where $A>0, \eta>0, M$ are constants to be determined. We have that

$$
\partial_{t} \bar{v}(x, t)=M\left[A e^{\eta t}+\eta h(x, t)\right] \quad\left(x \in \mathbb{R}^{N}, t \in[0, \delta]\right),
$$

whereas

$$
\begin{gathered}
-a(x)(-\Delta)^{s} \bar{v}(x, t)+c(x) \bar{v}(x, t)+f(x) \\
\leq M e^{\eta \delta} \max _{\bar{B}_{\delta}\left(x_{0}\right)}\left|a(-\Delta)^{s} \chi\right|+M c(x) h(x, t)+\|c\|_{\infty}\left(\left\|u_{0}\right\|_{\infty}+1\right)+\|f\|_{\infty} \quad\left(x \in \mathbb{R}^{N}, t \in[0, \delta]\right) .
\end{gathered}
$$

Therefore,

$$
\partial_{t} \bar{v}(x, t) \geq-a(x)(-\Delta)^{s} \bar{v}(x, t)+c(x) \bar{v}(x, t)+f(x) \quad\left(x \in \mathbb{R}^{N}, t \in[0, \delta]\right),
$$

if

$$
\eta \geq\|c\|_{\infty}, \quad A \geq \max _{\bar{B}_{\delta}\left(x_{0}\right)}\left|a(-\Delta)^{s} \chi\right|+\|c\|_{\infty}\left(\left\|u_{0}\right\|_{\infty}+1\right)+\|f\|_{\infty} .
$$

Furthermore, since

$$
h(x, t) \geq \delta^{2} \quad \text { for all } x \in \mathbb{R}^{N} \backslash B_{\delta}\left(x_{0}\right), t \in[0, \delta],
$$

it easily follows that

$$
\bar{v}(x, t) \geq u_{j}(x, t) \quad \text { for all } x \in \mathbb{R}^{N} \backslash B_{\delta}\left(x_{0}\right), t \in[0, \delta],
$$

if

$$
M \geq \frac{2 K_{T}}{\delta^{2}}
$$


From (4.11) we get

$$
\bar{v}(x, 0) \geq u_{j}(x, 0) \quad \text { for all } x \in B_{\delta}\left(x_{0}\right)
$$

while

$$
\bar{v}(x, 0) \geq M \delta^{2}+u_{0}\left(x_{0}\right) \geq u_{j}(x, 0) \text { for all } x \in \mathbb{R}^{N} \backslash B_{\delta}\left(x_{0}\right),
$$

due to (4.15).

Suppose that (4.13), (4.15) hold. Then, by (4.12), (4.14), (4.16), (4.17), for any $j \in \mathbb{N}, j>$ $R$ the function $\bar{v}$ is a supersolution (in the sense of Definition 2.2) of problem

$$
\begin{cases}\partial_{t} v=-a(-\Delta)^{s} v+c v+f & \text { in } B_{\delta}\left(x_{0}\right) \times(0, \delta] \\ v=u_{j} & \text { in }\left(\mathbb{R}^{N} \backslash B_{\delta}\left(x_{0}\right)\right) \times(0, \delta] \\ v=u_{j} & \text { in } \mathbb{R}^{N} \times\{0\},\end{cases}
$$

while $u_{j}$ is a solution of the same problem. By the comparison principle (see Remark 2.5) we obtain

$$
u_{j} \leq \bar{v} \quad \text { in } B_{\delta}\left(x_{0}\right) \times(0, \delta]
$$

Define

$$
\underline{v}(x, t):=-M h(x, t)+u_{0}\left(x_{0}\right)-\epsilon \quad\left(x \in \mathbb{R}^{N}, t \in[0, \delta]\right) ;
$$

suppose that (4.13) and (4.15) hold. By the same arguments as above, we can show that there holds

$$
u_{j} \geq \underline{v} \quad \text { in } B_{\delta}\left(x_{0}\right) \times(0, \delta] .
$$

Inequalities (4.19)-(4.20) yield

$$
-M h(x, t)-\epsilon \leq u_{j}(x, t)-u_{0}\left(x_{0}\right) \leq M h(x, t)+\epsilon
$$

for all $x \in B_{\delta}\left(x_{0}\right), t \in[0, \delta]$. Letting $j \rightarrow \infty$, thus we obtain

$$
-M h(x, t)-\epsilon \leq u(x, t)-u_{0}\left(x_{0}\right) \leq M h(x, t)+\epsilon
$$

for all $x \in B_{\delta}\left(x_{0}\right), t \in(0, \delta]$. Letting $x \rightarrow x_{0}, t \rightarrow 0^{+}$, and then $\epsilon \rightarrow 0^{+}$, we get that $\lim _{x \rightarrow x_{0}} u(x, t)=u_{0}\left(x_{0}\right)$. Hence the Claim 1 has been shown.

Claim 2: We have that

$$
\lim _{|x| \rightarrow \infty} u(x, t)=g(t) \quad \text { uniformly with respect to } t \in[0, T]
$$

In fact, fix any $\tau_{0} \in[0, T], 0<\epsilon<1$. Since $g \in C([0, T])$, there exists $\delta \in(0,1)$ such that

$$
g\left(t_{0}\right)-\epsilon \leq g(t) \leq g\left(t_{0}\right)+\epsilon \quad \text { for any } t \in\left[\underline{t}_{\delta}, \bar{t}_{\delta}\right]
$$

where

$$
\underline{t}_{\delta}:=\max \left\{t_{0}-\delta, 0\right\}, \quad \bar{t}_{\delta}:=\min \left\{t_{0}+\delta, T\right\} .
$$

Clearly, $\delta=\delta(\epsilon)$ does not depend on $t_{0}$. Furthermore, due to (1.3), there exists $R_{\epsilon}>0$ such that

$$
g(0)-\epsilon \leq u_{0}(x) \leq g(0)+\epsilon \text { for all } x \in \mathbb{R}^{N} \backslash B_{R_{\epsilon}} .
$$

Let $R \geq \max \left\{R_{0}, R_{\epsilon}\right\}$ with $R_{0}$ given by Proposition 3.1 set

$$
N_{j}^{R}:=B_{j} \backslash B_{R} \quad \text { for any } j>R .
$$

Define

$$
\underline{w}(x, t):=-M V(x) e^{\eta t}-\lambda\left(t-t_{0}\right)^{2}+g\left(t_{0}\right)-\epsilon \quad \text { for all }(x, t) \in \mathbb{R}^{N} \times[0, T]
$$


where $M>0, \eta>0, \lambda>0$ are constants to be chosen in the sequel, while $V(x) \equiv V(|x|)$ is the supersolution given by Proposition 3.1 .

In view of Proposition 3.1, we have $-a(x)(-\Delta)^{s} \underline{w}+c(x) \underline{w} \geq M e^{\eta t}-M c(x) V(x) e^{\eta t}-\|c\|_{\infty}\left(\|g\|_{\infty}+\lambda+1\right) \quad$ for all $x \in N_{j}^{R}, t \in[0, T]$.

Therefore,

$$
\begin{gathered}
\partial_{t} \underline{w}+a(-\Delta)^{s} \underline{w}-c \underline{w}-f \\
\leq-\eta M V e^{\eta t}-2 \lambda\left(t-t_{0}\right)-M e^{\eta t}+c M V e^{\eta t} \\
+\|c\|_{\infty}\left(\|g\|_{\infty}+\lambda+1\right)+\|f\|_{\infty} \leq 0 \quad \text { in } N_{j}^{R} \times(0, T],
\end{gathered}
$$

if we take

$$
\begin{gathered}
\eta \geq\|c\|_{\infty}, \\
M \geq 2 \lambda+\|f\|_{\infty}+\|c\|_{\infty}\left(\|g\|_{\infty}+\lambda+1\right)+\|f\|_{\infty} .
\end{gathered}
$$

In view of (4.8), we obtain

$$
\underline{w}(x, t) \leq-M V(R)+\|g\|_{\infty} \leq-K_{T} \leq u_{j}(x, t) \text { for all } x \in \bar{B}_{R}, t \in\left(\underline{t}_{\delta}, \bar{t}_{\delta}\right),
$$

if

$$
M \geq \frac{\|g\|_{\infty}+K_{T}}{V(R)}
$$

From (4.23) we have

$$
\underline{w}(x, t) \leq g(t) \quad \text { for all } x \in \mathbb{R}^{N} \backslash B_{j}, t \in\left(\underline{t}_{\delta}, \bar{t}_{\delta}\right) .
$$

Suppose that $\underline{t}_{\delta}=0$ (note that this is always the case when $t_{0}=0$ ). From (4.24) and (4.23)

$$
\underline{w}(x, 0) \leq g(0)-\epsilon \leq u_{j}(x, 0)=u_{0, j}(x) \quad \text { for all } x \in \mathbb{R}^{N} \backslash B_{R} ;
$$

while

$$
\underline{w}(x, 0) \leq-M V\left(R_{\epsilon}\right)+\|g\|_{\infty} \leq-K_{T} \leq u_{j}(x, 0) \text { for all } x \in B_{R},
$$

provided that (4.30) holds.

Suppose that $\underline{t}_{\delta}>0$. It follows from (4.8) that

$$
\underline{w}\left(x, \underline{t}_{\delta}\right) \leq-\lambda \delta^{2}+\|g\|_{\infty} \leq-K_{T} \leq u_{j}\left(x, \underline{t}_{\delta}\right) \quad \text { for all } x \in \mathbb{R}^{N}, t \in\left(\underline{t}_{\delta}, \bar{t}_{\delta}\right),
$$

if

$$
\lambda \geq \frac{\|g\|_{\infty}+K_{T}}{\delta^{2}} .
$$

Now, suppose that (4.27), (4.28), (4.30), (4.35) hold. By (4.26), (4.29), (4.31), (4.32), (4.33), (4.34), for any $j \in \mathbb{N}, j>R$, the function $\underline{w}$ is a subsolution (in the sense of Definition 2.2) of problem

$$
\begin{cases}\partial_{t} v=-a(-\Delta)^{s} v+c v+f & \text { in } N_{j}^{R} \times\left(\underline{t}_{\delta}, \bar{t}_{\delta}\right] \\ v=u_{j} & \text { in }\left(\mathbb{R}^{N} \backslash N_{j}^{R}\right) \times(0, T] \\ v=u_{j} & \text { in } \mathbb{R}^{N} \times\{0\},\end{cases}
$$

while $u_{j}$ is a solution of the same problem. By the comparison principle (see Remark 2.5) we obtain

$$
\underline{w} \leq u_{j} \quad \text { in } \quad N_{j}^{R} \times\left(\underline{t}_{\delta}, \bar{t}_{\delta}\right] .
$$


Define

$$
\bar{w}(x, t):=M V(x) e^{\eta t}+\lambda\left(t-t_{0}\right)^{2}+g\left(t_{0}\right)+\epsilon \text { for all } x \in \mathbb{R}^{N}, t \in[0, T] ;
$$

suppose that (4.27), (4.28), (4.30), (4.35). By the same arguments as above, we can show that there holds

$$
\bar{w} \geq u_{j} \quad \text { in } N_{j}^{R} \times\left(\underline{t}_{\delta}, \bar{t}_{\delta}\right] .
$$

From (4.37) and (4.39) we get

$$
-M V(x) e^{\eta t}-\lambda\left(t-t_{0}\right)^{2}-\epsilon \leq u_{j}(x, t)-g\left(t_{0}\right) \leq M V(x) e^{\eta t}+\lambda\left(t-t_{0}\right)^{2}+\epsilon
$$

for all $x \in N_{j}^{R}, t \in\left(\underline{t}_{\delta}, \bar{t}_{\delta}\right]$. Choosing $t=t_{0}$ in (4.40) and letting $j \rightarrow \infty$, we obtain

$$
-M V(x) e^{\eta T}-\epsilon \leq u\left(x, t_{0}\right)-g\left(t_{0}\right) \leq M V(x) e^{\eta T}+\epsilon \quad \text { for all } x \in \mathbb{R}^{N} \backslash B_{R} .
$$

From (4.41) it follows that

$$
\sup _{t_{0} \in[0, T]}\left|u\left(x, t_{0}\right)-g\left(t_{0}\right)\right| \leq \bar{C} V(x)+\epsilon \quad \text { for all } x \in \mathbb{R}^{N} \backslash B_{R},
$$

where $\bar{C}:=M e^{\eta T}$. Due to (4.42) and (1.12), letting $|x| \rightarrow \infty, \epsilon \rightarrow 0^{+}$, we obtain (1.4). Hence the Claim 2 has been shown.

Finally, this solution is unique, due to Proposition 4.1.

Now we prove Theorem 2.8 We follow the same line of arguments of the proof of Theorem 2.8. but there is an important difference. In fact, we need to substitute the estimate (4.8), which is dependent on $T$, by another one independent of $T$. In order to obtain such better estimate we use the supersolution $h$ constructed in Proposition 3.2 .

Proof of Theorem 2.8. Arguing as in the proof of Theorem 2.7 we construct the sequence $u_{j}(x, t)$ of solutions of problem (4.5) with $T=\infty$. Let $h(x)$ be the supersolution provided by Proposition 3.2. Then obviously

$$
V_{0}(x):=h(x)-\inf _{\mathbb{R}^{N}} h+1
$$

is also a supersolution of (1.16) and $V_{0}(x) \geq 1$. Let $B:=\max \left\{\|f\|_{\infty},\left\|u_{0}\right\|_{\infty},\|g\|_{\infty}\right\}$. Since $c \leq 0$, we have that $B V_{0}$ is a supersolution of problem (4.5), while $-B V_{0}$ is a subsolution of (4.5). Thus, by the comparison principle,

$$
\left|u_{j}\right| \leq B V_{0} \quad \text { in } \quad B_{j} \times(0, \infty) .
$$

Passing to the limit as $j \rightarrow \infty$ we obtain that

$$
|u| \leq B V_{0} \leq \check{C}:=B\left\|V_{0}\right\|_{\infty} \quad \text { in } \mathbb{R}^{N} \times(0, \infty) .
$$

Note that estimate (4.45) substitutes estimate (4.8) which is depending on $T$. Now, consider the functions $\underline{w}$ and $\bar{w}$ defined in (4.25) and in (4.38), respectively. Suppose that $\eta=0$,

$$
\lambda \geq \frac{\|g\|_{\infty}+\check{C}}{\delta^{2}}, M \geq \frac{\|g\|_{\infty}+\check{C}}{V(R)},
$$

and (4.28) holds. Note that $M$ and $\lambda$ do not depend on $T$. Since $c \leq 0$ and (4.45) holds, by the same arguments as in the proof of Theorem 2.7 we can infer that for any $\epsilon>0$

$$
\sup _{t_{0} \in[0, \infty)}\left|u\left(x, t_{0}\right)-g\left(t_{0}\right)\right| \leq M V(x)+\epsilon \quad \text { for all } x \in \mathbb{R}^{N} \backslash B_{R} .
$$

Thanks to (4.46) and (1.12), letting $|x| \rightarrow \infty, \epsilon \rightarrow 0^{+}$, we obtain (1.14). Finally, this solution is unique, due to Proposition 4.1. This completes the proof.

We have the next quite standard comparison principle. 
Proposition 4.2 Let assumptions $\left(H_{0}\right),\left(H_{1}\right)$ be satisfied. Suppose that $c \leq 0$ in $\mathbb{R}^{N}$. Let $u$ be a subsolution and $v$ a supersolution to equation (1.2) such that

$$
\limsup _{|x| \rightarrow \infty}(u-v) \leq 0 .
$$

Then

$$
u \leq v \quad \text { in } \mathbb{R}^{N} \text {. }
$$

Proof. Set $w:=u_{1}-u_{2}$. Let $\epsilon>0$. Then there exists $R_{\epsilon}>0$ such that

$$
|w(x)| \leq \epsilon \text { for all } x \in \mathbb{R}^{N} \backslash B_{R_{\epsilon}} .
$$

Hence $w$ is a subsolution of problem

$$
\begin{cases}-a(-\Delta)^{s} v+c v=0 & \text { in } B_{R_{\epsilon}} \\ v=\epsilon & \text { in } \mathbb{R}^{N} \backslash B_{R_{\epsilon}} .\end{cases}
$$

Moreover, it is easily seen that the function $z \equiv \epsilon$ is a supersolution of problem (4.47). So, by the comparison principle (see Remark 2.6),

$$
w \leq \epsilon \quad \text { in } \mathbb{R}^{N} .
$$

Similarly, it can be shown that

$$
w \geq-\epsilon \text { in } \mathbb{R}^{N}
$$

Letting $\epsilon \rightarrow 0^{+}$, we get $w=0$ in $\mathbb{R}^{N}$. Hence the proof is complete.

Now, we prove Theorem 2.10.

Proof of Theorem 2.10. Let $\gamma \in \mathbb{R}$. For any $j \in \mathbb{N}$ let $u_{j}$ be the unique solution (see Remark 2.6) of the problem

$$
\begin{cases}a(-\Delta)^{s} u-c u=f & \text { in } B_{j} \times(0, T] \\ u=\gamma & \text { in } \mathbb{R}^{N} \backslash B_{j}\end{cases}
$$

We claim that there exists $K>0$ such that for any $j \in \mathbb{N}$

$$
\left|u_{j}(x)\right| \leq K \text { for all } x \in \mathbb{R}^{N} .
$$

In fact, let $h=h(x) \equiv h(|x|)$ be the supersolution given by Proposition 3.2, Define

$$
\tilde{h}:=C(h+1) \text { in } \mathbb{R}^{N},
$$

where $C \geq \max \left\{\gamma,\|f\|_{\infty}\right\}$. It is easily seen that, for any $j \in \mathbb{N}, h$ is a supersolution of problem (4.50). Therefore, by the comparison principle (see Remark 2.6), we get (4.51), with $K=\|\tilde{h}\|_{\infty}$.

By the a priori estimates recalled in Remark 2.6 $(i i i)$ and usual compactness arguments, there exists a subsequence $\left\{u_{j_{k}}\right\} \subset\left\{u_{j}\right\}$ and a function $u \in C\left(\mathbb{R}^{N}\right)$ such that

$$
u:=\lim _{k \rightarrow \infty} u_{j_{k}} \text { uniformly in } D,
$$

for any compact subset $D \subset \mathbb{R}^{N}$. For simplicity, we still denote $\left\{u_{j_{k}}\right\}$ by $\left\{u_{j}\right\}$. In view of stability properties of viscosity solutions under local uniform convergence, the function $u$ is a solution of equation

$$
a(-\Delta)^{s} u-c u=f \quad \text { in } \mathbb{R}^{N} .
$$

Claim : The solution $u$ satisfies condition (1.21). 
In fact, define

$$
\underline{w}(x):=-M h(x)+\gamma \text { for all } x \in \mathbb{R}^{N},
$$

where $M>0$ is a constant to be chosen in the sequel.

In view of Proposition 3.2, it is easily seen that, if we take

$$
M \geq\|c\|_{\infty} \gamma+\|f\|_{\infty},
$$

then $\underline{w}$ is a supersolution of problem (4.50), for any $j \in \mathbb{N}$. By the comparison principle (see Remark 2.6),

$$
\underline{w} \leq u_{j} \quad \text { in } \mathbb{R}^{N} .
$$

On the other hand, by the same methods as above, we can show that

$$
u_{j} \leq \bar{w} \text { in } \mathbb{R}^{N},
$$

where

$$
\bar{w}(x):=M h(x)+\gamma \text { for all } x \in \mathbb{R}^{N} .
$$

From (4.53), (4.54) it follows that

$$
-M h+\gamma \leq u_{j} \leq M h+\gamma \quad \text { in } \mathbb{R}^{N} .
$$

Letting $j \rightarrow \infty$, in view of (1.17) we have that (2.4) holds. So, the Claim has been shown.

Finally, the uniqueness of the solution $u$ follows from Proposition 4.2 .

\section{Asymptotic behaviour of solutions: proofs}

To begin with, we show the next auxiliary result.

Proposition 5.1 Let assumptions of Theorem 2.8 be satisfied with $g \equiv g_{1}$. Assume that

$$
g_{1}\left(t_{1}\right) \leq g_{1}\left(t_{2}\right) \quad \text { for any } 0 \leq t_{1}<t_{2} .
$$

Let $\underline{V}:=-A V_{0}$, with

$$
A \geq\left\|g_{1}\right\|_{\infty}+\|f\|_{\infty}
$$

and $V_{0}$ defined in (4.43). Let $\underline{w}$ be the unique solution, provided by Theorem 2.8, of the problem

$$
\begin{cases}\partial_{t} u=-a(-\Delta)^{s} u+c u+f & \text { in } \mathbb{R}^{N} \times(0, \infty) \\ u=\underline{V} & \text { in } \mathbb{R}^{N} \times\{0\}\end{cases}
$$

such that

$$
\lim _{|x| \rightarrow \infty} \underline{w}(x, t)=g_{1}(t) \quad \text { uniformly for } t \in[0, \infty) .
$$

Then $t \mapsto \underline{w}(x, t)$ is nondecreasing, i.e.,

$$
\underline{w}\left(x, t_{1}\right) \leq \underline{w}\left(x, t_{2}\right) \quad \text { for all } x \in \mathbb{R}^{N}, 0 \leq t_{1}<t_{2} .
$$

Proof of Proposition 5.1. It is easily seen that $\underline{V}$ is a subsolution of problem (5.3) . In fact, since $c \leq 0$ and $\underline{V}<0$, due to (5.2) we have (in the viscosity sense)

$$
-a(-\Delta)^{s} \underline{V}+c \underline{V}+f \geq A-\|f\|_{\infty} \geq 0=\partial_{t} \underline{V} \quad \text { in } \mathbb{R}^{N} \times(0, \infty) .
$$

Moreover,

$$
\underline{V}-\underline{w}=0 \text { in } \mathbb{R}^{N} \times\{0\}
$$


and by (5.2) and (5.4),

$$
\limsup _{|x| \rightarrow \infty}[\underline{V}(x)-\underline{w}(x, t)] \leq 0 \quad \text { uniformly for } t \in[0, \infty) .
$$

Since $\underline{w}$ is a solution of problem (5.3), by Proposition 4.1 ,

$$
\underline{V}(x)=\underline{w}(x, 0) \leq \underline{w}(x, t) \text { for all } x \in \mathbb{R}^{N}, t>0 .
$$

In order to show (5.5), take any $t_{0}>0$ and define

$$
\tilde{w}(x, t):=\underline{w}\left(x, t+t_{0}\right) \text { for all } x \in \mathbb{R}^{N}, t>0 .
$$

Note that both $\underline{w}$ and $\tilde{w}$ satisfy the equation

$$
\partial_{t} v-a(-\Delta)^{s} v-c v=f \quad \text { in } \mathbb{R}^{N} \times(0, \infty) .
$$

Moreover, from (5.6) we obtain that

$$
\tilde{w}(x, 0) \geq \underline{w}(x, 0) \text { for all } x \in \mathbb{R}^{N} .
$$

In addition, due to (5.1),

$$
\lim _{|x| \rightarrow \infty}[\tilde{w}(x, t)-\underline{w}(x, t)]=\tilde{g}_{1}\left(t+t_{0}\right)-g_{1}(t) \geq 0 \quad \text { uniformly for } t \in[0, \infty) .
$$

Thus, by Proposition 4.1,

$$
\tilde{w}(x, t) \geq \underline{w}(x, t) \text { for all } x \in \mathbb{R}^{N}, t>0 .
$$

Hence the conclusion follows.

Similarly, we can show the next result.

Proposition 5.2 Let assumptions of Theorem 2.8 be satisfied with $g \equiv g_{2}$. Assume that

$$
g_{2}\left(t_{1}\right) \geq g_{2}\left(t_{2}\right) \quad \text { for any } 0 \leq t_{1}<t_{2} .
$$

Let $\bar{V}:=-A V_{0}$, where $V_{0}$ is defined in (5.9) and $A$ in (5.2) .

Let $\bar{w}$ be the unique solution, provided by Theorem 2.8, of the problem

$$
\begin{cases}\partial_{t} u=-a(-\Delta)^{s} u+c u+f & \text { in } \mathbb{R}^{N} \times(0, \infty) \\ u=\bar{V} & \text { in } \mathbb{R}^{N} \times\{0\}\end{cases}
$$

such that

$$
\lim _{|x| \rightarrow \infty} \bar{w}(x, t)=g_{2}(t) \quad \text { uniformly for } t \in[0, \infty) .
$$

Then $t \mapsto \bar{w}(x, t)$ is nonincreasing, i.e.,

$$
\bar{w}\left(x, t_{1}\right) \geq \bar{w}\left(x, t_{2}\right) \quad \text { for all } x \in \mathbb{R}^{N}, 0 \leq t_{1}<t_{2} .
$$

Now we prove the next result.

Proposition 5.3 Let assumptions of Theorem 2.8 be satisfied. Let $g_{1} \in C([0, \infty)) \cap L^{\infty}((0, \infty))$ with

$$
\begin{gathered}
g_{1}(t) \leq g(t) \quad \text { for all } t \in[0, \infty), \\
\lim _{t \rightarrow \infty} g_{1}(t)=\lim _{t \rightarrow \infty} g(t) ;
\end{gathered}
$$

suppose that (5.1) is satisfied. Let $\underline{w}$ be given by Proposition [5.1, also supposing that

$$
A \geq\left\|u_{0}\right\|_{\infty} .
$$

Then

$$
\underline{w}(x, t) \leq u(x, t) \quad \text { for all } x \in \mathbb{R}^{N}, t>0 .
$$


Proof. Let $z:=\underline{w}-u$. Note that $z$ solves equation

$$
\partial_{t} z=-a(-\Delta)^{s} z+c z \quad \text { in } \mathbb{R}^{N} \times(0, \infty) .
$$

In view of (5.14) we have

$$
z(x, 0)=\underline{V}(x)-u_{0}(x) \leq 0 \text { for all } x \in \mathbb{R}^{N} .
$$

Moreover, from (5.12) we obtain

$$
\lim _{|x| \rightarrow \infty} z(x, t)=g_{1}(t)-g(t) \leq 0 \quad \text { uniformly for } t \in[0, \infty) .
$$

Hence, by Proposition 4.1 ,

$$
z \leq 0 \text { for all } x \in \mathbb{R}^{N}, t>0
$$

This completes the proof.

Analogously to Proposition 5.3 , the next result can be shown.

Proposition 5.4 Let assumptions of Theorem [2.8 be satisfied. Let $g_{2} \in C([0, \infty)) \cap L^{\infty}((0, \infty))$ with

$$
\begin{gathered}
g_{2}(t) \geq g(t) \quad \text { for all } t \in[0, \infty), \\
\lim _{t \rightarrow \infty} g_{2}(t)=\lim _{t \rightarrow \infty} g(t) ;
\end{gathered}
$$

suppose that (5.8) is satisfied. Let $\bar{w}$ be given by Proposition [5.2, also supposing that (5.14) holds. Then

$$
\bar{w}(x, t) \geq u(x, t) \quad \text { for all } x \in \mathbb{R}^{N}, t>0 .
$$

Now we are in position to prove Theorem 2.11.

Proof of Theorem 2.11. Keep the same notation as in Propositions 5.155.4 In view of (5.5) and (5.11), we can define

$$
\underline{W}(x):=\lim _{t \rightarrow \infty} \underline{w}(x, t), \quad \bar{W}(x):=\lim _{t \rightarrow \infty} \bar{w}(x, t) \quad \text { for any } x \in \mathbb{R}^{N} .
$$

Observe that the constant $C$ in Remark 2.5 do not depend on $T$, since $a, c, f$ does not depend on $t$. Consequently we have that $\underline{w} \rightarrow \underline{W}, \bar{w} \rightarrow \bar{W}$ as $t \rightarrow \infty$ uniformly in each compact subset of $\mathbb{R}^{N}$; thus, $\underline{W}, \bar{W} \in C\left(\mathbb{R}^{\bar{N}}\right)$. We claim that both $\underline{W}$ and $\bar{W}$ solve

$$
a(-\Delta)^{s} u-c u=f \quad \text { in } \mathbb{R}^{N} .
$$

In fact, we limit ourselves to show that $\underline{W}$ is a subsolution of equation (5.20), since the remaining part of the claim follows analogously.

Now, let $\left\{t_{n}\right\} \subset(0, \infty)$ be a sequence with $t_{n} \rightarrow \infty$ as $n \rightarrow \infty$. Set

$$
\underline{w}_{n}(x):=\underline{w}\left(x, t_{n}\right) \quad\left(x \in \mathbb{R}^{N}\right) .
$$

Thus, $\underline{w}_{n} \rightarrow \underline{W}$ locally uniformly in $\mathbb{R}^{N}$ as $n \rightarrow \infty$.

Take any bounded subset $U \subset \mathbb{R}^{N}, x_{0} \in U$, take any test function $\varphi \in C^{2}\left(\mathbb{R}^{N}\right)$ such that

$$
\underline{W}\left(x_{0}\right)-\varphi\left(x_{0}\right) \geq \underline{W}(x)-\varphi(x) \text { for all } x \in U .
$$

Choose $\xi \in C^{2}\left(\mathbb{R}^{N}\right)$ with

$$
0 \leq \xi<1 \quad \text { if } x \in \mathbb{R}^{N} \backslash\left\{x_{0}\right\}, \xi\left(x_{0}\right)=1 .
$$


Fix any $\epsilon>0$. So,

$$
\underline{W}\left(x_{0}\right)-\left[\varphi\left(x_{0}\right)-\epsilon \xi\left(x_{0}\right)\right]>\underline{W}(x)-[\varphi(x)-\epsilon \xi(x)] \text { for all } x \in U \backslash\left\{x_{0}\right\} .
$$

It is easily seen that there exists $\bar{n}=\bar{n}(\epsilon) \in \mathbb{N}$ such that for any $n>\bar{n}$, for some $x_{n}^{\epsilon} \in U$,

$$
\underline{w}_{n}\left(x_{n}^{\epsilon}\right)-\left[\varphi\left(x_{n}^{\epsilon}\right)-\epsilon \xi\left(x_{n}^{\epsilon}\right)\right] \geq \underline{w}_{n}(x)-[\varphi(x)-\epsilon \xi(x)] \text { for all } x \in U ;
$$

moreover, for each $\epsilon>0, x_{n}^{\epsilon} \rightarrow x_{0}$ as $n \rightarrow \infty$.

Since $\underline{w}$ is a solution of (5.3), due to Definition 2.1] we have that

$$
0=\partial_{t} \chi\left(x_{n}^{\epsilon}\right) \leq-a\left(x_{n}^{\epsilon}\right)(-\Delta)^{s} \chi\left(x_{n}^{\epsilon}\right)+c\left(x_{n}^{\epsilon}\right) \underline{w}_{n}\left(x_{n}^{\epsilon}\right)+f\left(x_{n}^{\epsilon}\right),
$$

with

$$
\chi \equiv \chi_{\epsilon, n}:= \begin{cases}\varphi-\epsilon \xi & \text { in } U \\ \underline{w}_{n} & \text { in } \mathbb{R}^{N} \backslash U .\end{cases}
$$

Note that

$$
(-\Delta)^{s} \chi\left(x_{n}\right)=C_{N, s}\left\{\int_{U} \frac{\varphi\left(x_{n}^{\epsilon}\right)-\epsilon \xi\left(x_{n}^{\epsilon}\right)-[\varphi(y)-\epsilon \xi(y)]}{\left|x_{n}^{\epsilon}-y\right|^{N+2 s}} d y+\int_{\mathbb{R}^{N} \backslash U} \frac{\underline{w}_{n}\left(x_{n}^{\epsilon}\right)-\underline{w}(y)}{\left|x_{n}^{\epsilon}-y\right|^{N+2 s}} d y\right\} .
$$

Since $\varphi, \chi \in C^{2}(U)$, for any $\epsilon>0$ we have

$$
\lim _{n \rightarrow \infty} \int_{U} \frac{\varphi\left(x_{n}^{\epsilon}\right)-\epsilon \xi\left(x_{n}^{\epsilon}\right)-[\varphi(y)-\epsilon \xi(y)]}{\left|x_{n}^{\epsilon}-y\right|^{N+2 s}} d y=\int_{U} \frac{\varphi\left(x_{0}\right)-\varphi(y)}{\left|x_{0}-y\right|^{N+2 s}} d y+\epsilon \int_{U} \frac{\xi(y)-\xi\left(x_{0}\right)}{\left|x_{0}-y\right|^{N+2 s}} d y
$$

furthermore,

$$
\lim _{n \rightarrow \infty} \int_{\mathbb{R}^{N} \backslash U} \frac{\underline{w}_{n}\left(x_{n}^{\epsilon}\right)-\underline{w}_{n}(y)}{\left|x_{n}^{\epsilon}-y\right|^{N+2 s}} d y=\int_{\mathbb{R}^{N} \backslash U} \frac{\underline{W}\left(x_{0}\right)-\underline{W}(y)}{\left|x_{0}-y\right|^{N+2 s}} d y .
$$

From (5.23), (5.24), (5.25), letting $n \rightarrow \infty$ in (5.22), we have, for any $\epsilon>0$,

$$
0 \leq-a\left(x_{0}\right)(-\Delta)^{s} \psi\left(x_{0}\right)-a\left(x_{0}\right) \epsilon \int_{U} \frac{\xi(y)-\xi\left(x_{0}\right)}{\left|x_{0}-y\right|^{N+2 s}} d y+c\left(x_{0}\right) \underline{w}\left(x_{0}\right)+f\left(x_{0}\right),
$$

with

$$
\psi:= \begin{cases}\varphi & \text { in } U \\ \underline{W} & \text { in } \mathbb{R}^{N} \backslash U .\end{cases}
$$

Letting $\epsilon \rightarrow 0$, the claim follows.

Note that, in view of (5.4), (5.10), (5.13), (5.17), we can infer that

$$
\lim _{|x| \rightarrow \infty} \underline{W}(x)=\bar{W}(x)=\gamma,
$$

where $\gamma=\lim _{t \rightarrow \infty} g(t)$. By Proposition 4.2,

$$
\underline{W}(x)=\bar{W}(x) \text { for all } x \in \mathbb{R}^{N} .
$$

By (5.15) and (5.18),

$$
\underline{w}(x, t) \leq u(x, t) \leq \bar{w}(x, t) \text { for all } x \in \mathbb{R}^{N}, t>0 .
$$

Letting $t \rightarrow \infty$, due to (5.19) and (5.26), we get the thesis, with $W:=\underline{W} \equiv \bar{W}$. 


\section{References}

[1] H. Abels, M. Kassmann, The Cauchy problem and the martingale problem for integrodifferential operators with non-smooth kernels, Osaka J. Math., 46 (2009), 661-683.

[2] D.G. Aronson, P. Besala, Uniqueness of solutions to the Cauchy problem for parabolic equations, J. Math. Anal. Appl. 13 (1966), 516-526.

[3] G. Barles, E. Chasseigne, C. Imbert, On the Dirichlet Problem for Second-Order Elliptic Integro-Differential Equations, Indiana Univ. Math. J. 57 (2008), 213-146.

[4] G. Barles, E. Chasseigne, C. Imbert, Holder continuity of solutions of second-order non-linear elliptic integro-differential equations, J. Eur. Math. Soc. 13 (2010), 1-26.

[5] R. F. Bass, "Stochastic Processes", Cambridge Series in Statistical and Probabilistic Mathematics, (2011)

[6] R.M. Blumenthal, R.K. Getoor, Some theorems on stable processes, Trans. Amer. Math. Soc. 95 (1960), 263-273.

[7] E. Di Nezza, G. Palatucci, E. Valdinoci, Hitchhiker's guide to the fractional Sobolev spaces, Bull. Sci. Math. 136 (2012), 521-573.

[8] S. D. Eidelman, S. Kamin and F. Porper, Uniqueness of solutions of the Cauchy problem for parabolic equations degenerating at infinity, Asympt. Anal., 22 (2000), 349-358.

[9] F. Ferrari, I. E. Verbitsky, Radial fractional Laplace operators and hessian inequalities, J. Diff. Eq. 253 (2012), 244-272 .

[10] R. K. Getoor, First passage times for symmetric stable processes in space, Trans. Amer. Math. Soc. 101(1961), 75-90.

[11] A. Grigoryan, Analytic and geometric background of recurrence and non-explosion of the Brownian motion on Riemannian manifolds, Bull. Amer. Math. Soc. 36 (1999), $135-249$.

[12] G. Grillo, M. Muratori, F. Punzo, Conditions at infinity for the inhomogeneous filtration equation, Ann. I. H. Poincaré-AN 31 (2014), 413-428

[13] G. Grillo, M. Muratori, F. Punzo, On the asymptotic behaviour of solutions to the fractional porous medium equation with variable density, Discr. Cont. Dyn. Syst.-A (to appear)

[14] G. Grillo, M. Muratori, F. Punzo, Weighted fractional porous media equations: existence and uniqueness of weak solutions with measure data, arXiv: 1312.6076, submitted (2014)

[15] A. M. Il'in, A. S. Kalashnikov, O. A. Oleinik, Linear equations of the second order of parabolic type, Russian Math. Surveys 17 (1962), 1-144.

[16] S. Kamin, M.A. Pozio, A. Tesei, Admissible conditions for parabolic equations degenerating at infinity, St. Petersburg Math. J. 19 (2008), 239-251.

[17] S. Kamin, F. Punzo, Dirichlet conditions at infinity for parabolic and elliptic equations, preprint (2015) .

[18] S. Kamin, F. Punzo, Prescribed conditions at infinity for parabolic equations, Comm. Cont. Math. 17 (2015).

[19] R. Mikulevicius, H. Pragarauskas, On the Cauchy problem for integro-differential operators in Holder classes and the uniqueness of the martingale problem, Potential Anal. 40 (2014), 539-563.

[20] R. Mikulevicius, H. Pragarauskas, On the Cauchy problem for integro-differential operators in Sobolev classes and the martingale problem, J. Diff. Eq. 256 (2014), 1581-1626.

[21] F.W.J. Olver, D. W. Lozier, R.F. Boisvert, C.W. Clark (eds.), "NIST Handbook of Mathematical functions", Cambridge University Press, New York, NY, 2010, available on-line at http://dlmf.nist.gov. 
[22] Y. Pinchover, On uniqueness and nonuniqueness of the positive Cauchy problem for parabolic equations with unbounded coefficients, Math.Z. 223 (1996), 569-586.

[23] F. Punzo, G. Terrone, On the Cauchy problem for a general fractional porous medium equation with variable density, Nonlin. Anal. 8 (2014), 27-47.

[24] F. Punzo, A. Tesei, Uniqueness of solutions to degenerate elliptic problems with unbounded coefficients, Ann. Inst. H. Poincaré (C) Non Linear Analysis, 26 (2009), 20012024 .

[25] F. Punzo, E. Valdinoci, Uniqueness in weighted Lebesgue spaces for a class of fractional parabolic and elliptic equations, J. Diff. Eq. 258 (2015) 555-587.

[26] D.H. Sattinger, Monotone Methods in Nonlinear Elliptic and Parabolic Boundary Value Problems, Indiana Univ. Math. J., 21, 979-1000 (1972).

[27] L. Silvestre, "Regularity of the obstacle problem for a fractional power of the Laplace operator" (PhD Thesis). The University of Texas at Austin (2005).

[28] A. N. Tihonov, Théorèmes d'unicité pour l'équation de la chaleur, Mat. Sb. 42 (1935), 199-215. 